\title{
A Systems Approach toward the Functional Connections of Attachment and Fear
}

\author{
Norbert Bischof
}

California Institute of Technology and Max Planck Institute for Behavioral Physiology

\begin{abstract}
Bischof, NonBert. A Systems Approach toward the Functional Connections of Attachment and Fear. Crmud Development, 1975, 46, 801-817. Proceeding from J. Bowlby's attachment theory and W. A. Mason's arousal theory, a control model is developed that is capable of accounting for basic processes of mammalian social behavior. In particular, the model simulates the transition from a state of attachment to familiar conspecifics and fear of strangers to a stage of detachment from the familiar and exploration of the stranger. Scme of the model's predictions were tested in animal experiments and proved to be in satisfactory correspondence with the behavior actually observed.
\end{abstract}

All social mammals, including man, discriminate between familiar and alien members of their own species. In early childhood, the familiar conspecific is an object of attachment. Losing him causes distress. The stranger, on the other hand, evokes wariness or even fear. Although much more can be said about that, this is the basic pattern.

Later in development, usually culminating around puberty, this pattern changes. The familiar partner turns less attractive, even repugnant, whereas the stranger becomes a fascinating object of exploratory approaches. These approaches may establish a new familiarity that again entails attachment. The newly formed ties recapitulate to some degree early childhood attachment-as is indicated by such phenomena as baby language exchanged between lovers or, more seriously, the symptoms of anxiety, distress, and depression upon separation or loss of the partner. The original attachment objects of childhood, although remaining familiar, do not normally regain a comparably high intimacy.

It is the first, early infant period of this sequence that is traditionally dealt with in developmental psychology. But, obviously, this period is only part of an integrative pattern meant to provide security and support to an individual throughout a lifetime and neverthe- less prevent inbreeding. It is questionable whether the part can be fully understood without having the whole in mind.

The present study attempts to draft a causal network of interacting observable and hypothetical factors that can plausibly be thought to underlie the processes outlined above. The method used is a modified version of systems analysis as it is applied in control engineering.

Except for an illustrating example (see fig. 9), this study does not introduce new experimental results. It intends to help organize the material that has already accumulated and to ask fruitful questions for future research. A certain level of abstraction and generality will be maintained. As matters stand, there is no point in constructing an explanation for every petty detail experimenters may have unearthed to date. We want to understand the basic structure, if possible. Moreover, there is a practical limit in effect; one cannot easily test a model that encompasses 50 or so independent parameters.

When engaging in a systems approach that includes attachment behavior in its scope, we are obviously bound to start from Bowlby's theory (1971). However, although this theory is creditable as a pioneering work, it should be regarded as a first step that requires elabo-

Author's current address: University of Zurich, Institute of Psychology, CH-8044 Zurich, Zürichbergstrasse 43, Switzerland.

[Child Development, 1975, 46, 801-817. (C) 1975 by the Society for Research in Child Development, Inc. All rights reserved.] 
ration in several respects. In particular, the phenomenon of fear does not seem to have a well-defined place in Bowlby's control model, and here it might be fruitful to combine Bowlby's ideas with others, such as those of Mason (1965). It is the aim of the present study to outline such a compound model.

\section{The Security System}

Attachment.-Bowlby proceeds from the uncontestable premise that continuous proximity to an appropriate care giver is crucial for the survival of a mammalian infant. If the infant started seeking his mother only when he was hungry or in acute danger, his effort might easily come too late. Thus, there was probably a selection pressure enforcing the development of an infantile propensity to stay in continuous contact with the care giver.

In altricial animals, to be sure, the newborn infant on his own may not yet be able to maintain proximity to his mother. The primary effort here is made by the mother, except for the role of behaviors such as distress calls emitted by the child. Later in infancy, however, the proximity-seeking activity is fully shared, or even taken over, by the child. This activity Bowlby refers to as attachment behavior.

Attachment behavior is elicited by a too distant and too prolonged withdrawal of the mother, and it is facilitated by additional stimuli that are likely to arouse fear. Attachment behavior has several components. One of these is the emission of signals that are likely to attract the mother's attention. The most commonly encountered signals of this kind are distress calls. Even more basic for attachment is approach behavior, such as seeking the mother, moving toward her, or following her. Some further expressions of attachment behavior (e.g., sucking, babbling, smiling, and laughing) are functionally more intricate and, though important, are ignored in the present analysis.

Bowlby conceives of the causal connections between the above-mentioned behavioral units as a system of "goal-corrected behavior." However, since he does not give an exact formalization of the feedback loop postulated, the predictions of his model are difficult to determine. The system of attachment behavior, even in its simplest form, is too complex for a pure verbal description. Hence I try to map it in terms of a control chart.

Security.-The intensity of attachment behavior may differ considerably between individuals and in the same individual at different times as well as at different stages of his development. Ainsworth has proposed the concept of "security of attachment" to account for some of these differences (Ainsworth \& Wittig 1969; cf. Bowlby 1971, p. 401). This seems to be a useful concept, all the more so since other authors dealing with attachment quite commonly talk about the mother as a source of infantile "security." It should be noted, however, that this term does not clearly distinguish between an emotional state (feeling more or less secure) and an environmental fact (the degree to which a given mother is capable of influencing this state). To be consistent, I use the term in the former sense only. Thus, by security I denote a hypothetical intraorganismic variable which increases with the proximity and the quality of the care giver.

Dependency.-Differences in attachment behavior cannot, however, be explained fully by the mother being more or less remote or "good." There is also, for the child, a differing need of being reassured. Thus, we must allow for an internal reference variable specifying a set point for security at a given time. This variable may be labeled as the degree of dependency felt by a child. Again, "dependency" does not denote the objective fact of actually being dependent on other people's care but, rather, the subjective state of feeling so.

The concept of dependency has been introduced into developmental psychology in connection with efforts to operationalize psychoanalytic theory (Sears, Rau, \& Alpert 1966; Sears, Whiting, Nowlis, \& Sears 1953). I use this concept in essentially the same sense as these authors, except that I understand it as a hypothetical construct rather than confining myself to the operational level. This seems to be legitimate since the control charts discussed below should provide a sufficiently precise nomological net to prevent vagueness or ambiguity.

Recent attempts have been made to clarify the distinction between the concepts of dependency and of attachment (cf. Bowlby, in press; Gewirtz 1972). There appears to be one main formal difference in that attachment is understood to be directed toward a specific 
object whereas dependency is not. In accordance with this, my model introduces dependency as a scalar variable. "Attachment" behavior will be evinced to belong to a group of variables that must be defined as vectors (discussed below).

It ought to be added that in present psychological and psychiatric usage "dependency" has acquired a slightly pejorative connotation. It is frequently understood to refer to certain deviant states of excessive helplessness that would more appropriately be termed "overdependency" (cf. Bowlby, in press). My defnition of "dependency," as of all other concepts used in this study, is strictly nonvaluational.

Appetence for security.-Figure 1 represents the model in its primary version. The mother organism $(M)$ has three groups of properties bearing on the infant's (I) behavior. It has an individual physiognomy that allows the child to identify it, it emits such communicative signals as smiling or vocalizing, and it is in a certain state of localization $\left(\operatorname{Loc}_{\boldsymbol{M}}\right)$. Information about these properties is received and processed by sensory and brain structures comprised within a block labeled detector. According to this information, the detector controls the general level of security felt by the infant. This security level is matched against the degree of dependency. Dependency is conceived of as a "free input" variable, that is, as a variable that is controlled by (endogenous and environmental) factors not specified in the model. If dependency exceeds security, the difference between both

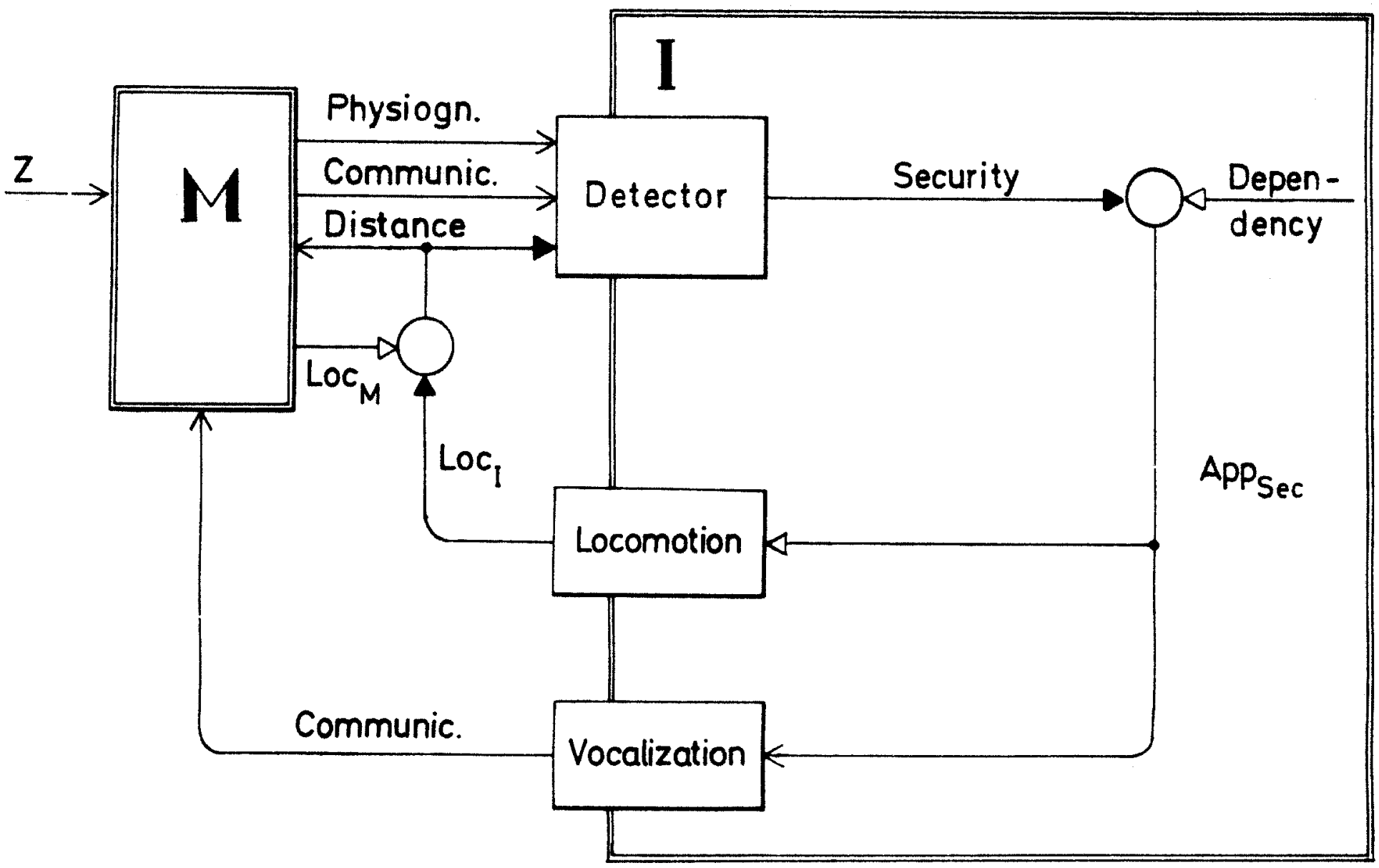

FIG. 1.-Security system, primary version. Symbols used in this and/or subsequent figures: Arrow = variable (scalar or vector). Block = system or subsystem in charge of processing sensory and/or programming motor variables. Orientation of arrowhead indicates direction of causation: arrows pointing toward block ("inputs") act on arrows originating in that block ("outputs"). Arrows originating (or terminating) in open space $=$ variables with causes (or effects) not specified by the model. Branchings ("soldered joints") are used when a given variable acts on the outputs of more than one block. Open triangular arrowhead = conservation of sign (positive correlation with output). Solid triangular arrowhead $=$ inversion of sign (negative correlation with output). Two-line arrowheads = no specification of sign. Open circle = mathematical operation resembling addition (or, in case of solid arrowhead, subtraction). Small open square (figs. $3,5,6)=$ operation resembling multiplication. Double line = border of organism. Variables enclosed by double lines (in figs. $3,5,6$, variables to right of double line) are hypothetical constructs; other variables are observables. $I=$ infant, $M=$ mother. Variables and subsystems interior to $M$ are not specified. Among variables determining $M$ 's behavior only distance of $I$ and communicative signals emitted by $I$ appear separately. All other factors influencing $M$ are comprised in one symbolic arrow, $Z$. 


\section{Child Development}

variables will be experienced as an appetence for security (Appsoc), and this appetence will release two groups of behavioral responsesnamely, a change of location $\left(\operatorname{Loc}_{I}\right)$ directed toward $M$, and the emission of communicative signals that motivate $M$ to change her location by an approach toward $I$. The difference between the locations of $M$ and $I$ defines (in terms of vector geometry) the distance of both, and the latter variable directly affects the detector. Decreasing distance will increase security; hence, there is a symbol of sign inversion (solid arrowhead) at the arrow denoting distance in the diagram.

Imprinting.-The degree of security engendered by a mother figure depends not only on her and the infant's present behavior but also on previous interactions between the two individuals, especially at the time of priming of the detector in figure 1. Clearly, at the onset of life the child cannot be aware of the mother's physiognomy. At best, his inborn information could concern general features of a female conspecific, although as a rule stimuli releasing a primal following response are much simpler. Only subsequently, in a rapid learning process, is the detector sensitized to the specific features of the one or few care givers who happen to be associated with the particular infant. This tuning of a detector for conspecific familiarity appears to be the essential feature of what is called "filial imprinting" by ethologists (e.g., Bateson 1966).

The proper imprinting of the detector may be supported by the mother's care-giving behavior or counteracted by her negligence. The latter would result in the mother's physiognomy later being unqualified to engender sufficient security in the infant; he would then neither accept her nor show signs of relaxation in her proximity. This is the other possible meaning of the term "security of attachment" as used by Ainsworth.

Surfeit.-Bowlby conceives of the security system as a negative feedback loop, which it certainly is. Everything else being equal, an increasing distance between mother and child would release a behavior appropriate to reduce this distance. What happens, however, if the deviation of security from its reference is in the other direction-that is, if there is an excess of security? Among technical feedback systems there are such gadgets as a refrigerator that turns on only when the temperature deviates from its set point in one direction, whereas it remains inactive if deviations occur in the opposite direction. On the other hand, we have thermostats that cause not only cooling of the room when the temperature rises but also heating when it falls. Bowlby thinks in terms of the first-mentioned, unidirectional type of process, when describing attachment behavior as "governed by a system that remains inactive as long as mother is in sight or in touch but that is apt to become activated when those conditions change. Once it is activated, approach continues .... until such time as the child is again within sight or touch of his mother, whereupon the system is terminated" (1971, p. 305). Bowlby extends this view even to later stages of ontogeny. Although he mentions certain adolescents "who cut themselves off from parents," he tends to regard them as slightly pathological extreme cases, whereas his ideal norm of a youth is one "whose attachment to parents remains strong" while, in addition, he forms equally strong ties to others (1971, p. 255).

Contrary to this notion, the study of animal social behavior (Bischof 1972a, 1972b, 1975a), as well as experience with human children (Rheingold \& Eckerman 1970), seems to indicate that the control system in question is in fact bidirectional. The appetence for security, that is, may turn negative and become equivalent to what Craig (1918) proposed to call an "aversion." In search of autonomy and identity, a juvenile would at least temporarily prefer to maintain a marked distance from the familiar surroundings of his childhood. In a state of low dependency, as could well be typical for puberty, the normal way to respond to the suffocating proximity of well-meaning attachment objects is withdrawal and the emission of signals discouraging continued approach of the former care giver.

This kind of behavior, which plays an important role in the prevention of inbreeding, can hardly be understood in terms of any theory that assigns all kinds of avoidance behavior to fear. I have proposed to call it surfeit response (Bischof 1975a) to point out that the object of withdrawal is a stimulus that under conditions of higher dependency would be, and has been, an object of attachment.

"Elastic-band"- and "magnet"-type attraction.-The concept of a control mechanism of attachment necessitates a further consideration. Normally, the feedback effecting the homeo- 
stasis of a given variable has to be "negative." In the case of attachment behavior, that is, the activities tending to reduce the distance between child and mother should intensify when this distance becomes larger, like an elastic band that exerts more force, the more it is expanded. Qualitatively, a plot resembling figure $2 a$ should ensue. This diagram does apply to some measures of attachment behavior-for instance, to the probability and intensity of distress signals. But the majority of criteria, including approach behavior, follow a different type of function (see fig. 2b). Under certain conditions these responses may even look like figure $2 c$, where

(a)



D

(b)
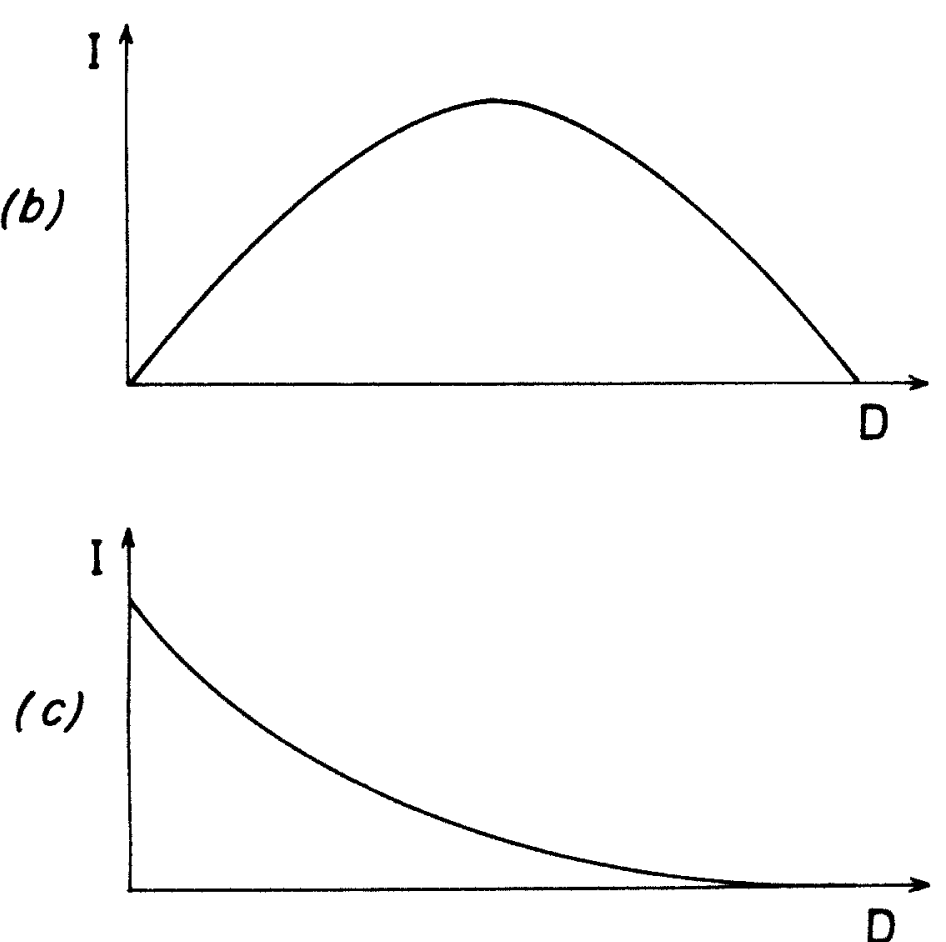

Fig. 2.-Three types of attraction by an object. $D=$ distance from object; $I=$ intensity of motivation toward affiliative contact with object. $a$, Elastic-band-type attraction-affliative motivation increases with distance until it reaches a maximum level that cannot be exceeded for physiological reasons (e.g., distress calls). b, Mixedtype attraction-at near range intensity of motivation varies according to $a$, whereas at larger distance it resembles $c$ (e.g., approach locomotion). $c$, Magnet-type attraction-affliative motivation increases with proximity (e.g., smiling response). approach-seeking behavior increases with proximity, as if due to the attraction of a magnet. Thus, a given response may produce consequences likely to enhance this response. This is called positive feedback, which by itself has no homeostatic properties.

Nevertheless, such a way of responding can be of survival value. Suppose the individual has two or more attachment objects, which for some reason happen to be spaced rather remotely from each other. If they do in fact exert an elastic-band influence on the child, he will-like Buridan's donkey-come to rest somewhere between them, without receiving care and shelter from any of them. If, however, they attract him like magnets, the place right between them will be of only labile equilibrium, and eventually he will join one of them. In experiments with human and animal young, the latter behavior is usually observed.

Incentive vector.-How, then, can this magnet-type behavior be accounted for in the framework of the negative-feedback model of attachment? Attachment behavior is based on two presuppositions: first, an appetence for security must be felt; second, stimuli must be available that orient locomotion toward a source of security.

The appetence component can parsimoniously be regarded as a one-dimensional or scalar quantity, a tension, varying only in intensity. This intensity can further be assumed to increase with distance in elastic-band fashion according to figure $2 a$.

The orientation component, on the other hand, must be conceived of as a vector: it has two dimensions-namely, a direction and an amount. The direction points to the mother. The amount has at least two states: zero for the mother being undetectable, and unity otherwise. But we can reasonably assume that the amount can vary in even finer graduations according to how clearly and unambiguously the mother can be identified and felt to be present. Plotted against distance, this function would then roughly resemble figure $2 c$.

Thus, the motivational force underlying attachment behavior is more complex than is indicated by figure 1 , in that we have to apply the old Hullian distinction between "drive" and "incentive strength." The former, equivalent to the appetence for security (Appsec), is a scalar quantity increasing with distance; 


\section{Child Development}

the latter, denotable as the incentive strength of the security object $\left(\operatorname{Inc}_{\mathrm{Sec}}\right)$, is a vector and increases with proximity.

Impulse vector.-Figure 3 represents the refined version of the security model. It assumes that appetence and incentive strength interact in a multiplicative fashion. This implies mainly that the common output of the two components is expected to vanish with either one of them being zero. Hence, if these components vary with distance according to figures $2 a$ and $2 c$, respectively, their product should follow figure $2 b$. This product, again, is a vector; it controls the intensity and orientation of overt attachment (or, if pointing away from the object, surfeit) behavior. I shall refer to this vector as the impulse of the security system $\left(\operatorname{Imp}_{\mathrm{Sec}}\right)$.

It may be noticed that figure $2 a$ could be computed by subtracting the curve in figure $2 c$ from a horizontal straight line. The curve in figure $2 a$, as stated earlier, represents the appetence for security. Dependency, as an endogenous or at least inert variable, is not contingent on the momentary distance of the mother and therefore indeed appears as a horizontal line when plotted against distance. Security can be supposed to increase with proximity; it should therefore have a characteristic curve similar to that of figure $2 c$. All this is in good accordance with the initial assumption, incorporated in figure 1 , that the

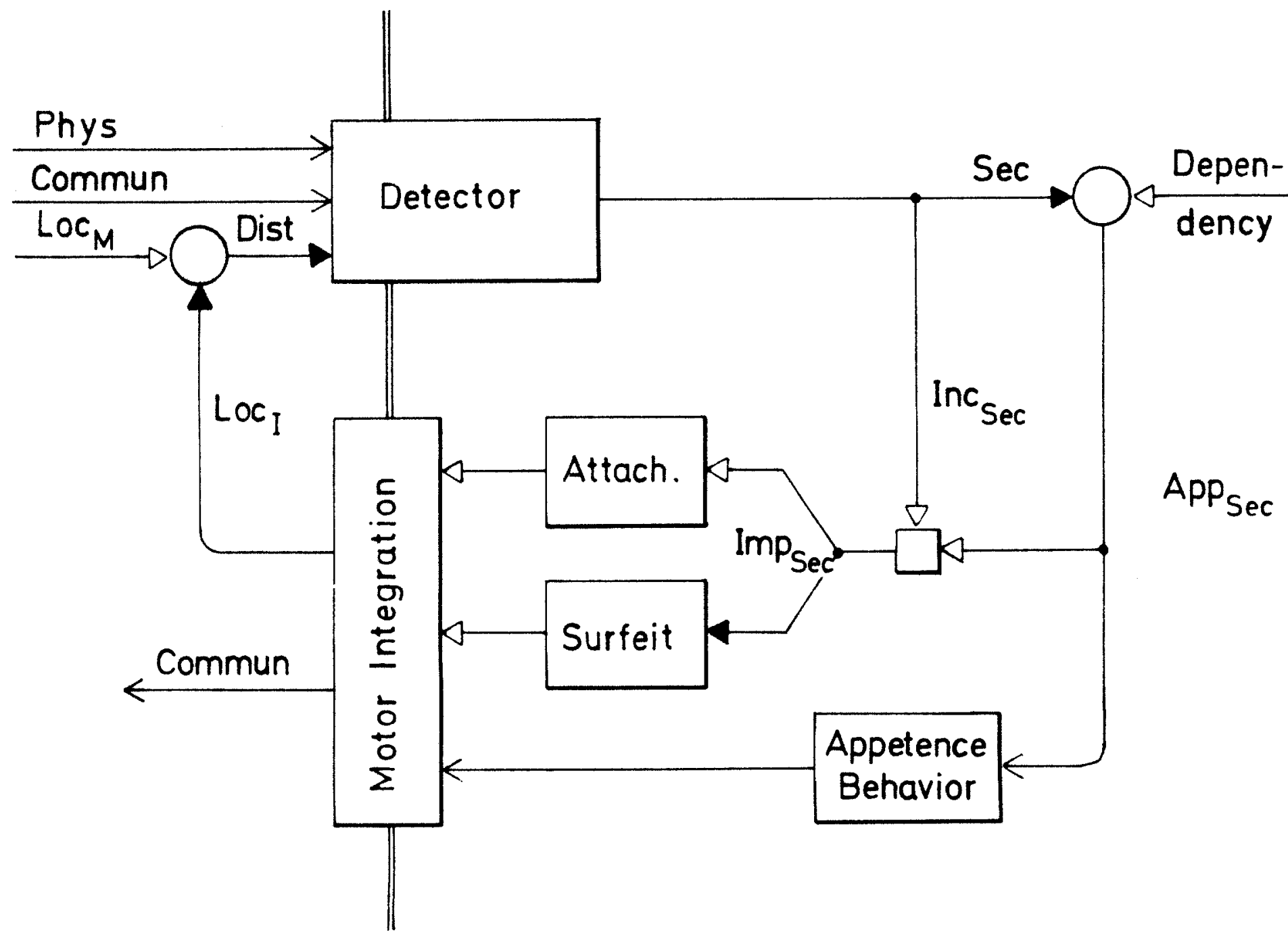

Frc. 3.-Security system, modified version. Mother object $M$ and all inputs of $M$ are neglected. Output of detector is a vector oriented toward $M$ and denoting her incentive strength as a security provider $\left(\operatorname{Inc}_{\mathrm{Sec}}\right.$ ). The model conceives the (scalar) magnitude of this strength to be equal to the security (Sec) actually effected by $M$ at this given distance. When Sec is subtracted from dependency, the difference, called appetence for security $\left(\mathrm{App}_{\mathrm{Sec}}\right)$, defines the drive component of the security system. The product of $\mathrm{App}_{\mathrm{Sec}}$ and $\mathrm{Inc}_{\mathrm{Sec}}$ is equivalent to the locomotor impulse exerted by the security system ( $\mathrm{Imp}_{\mathrm{Sec}}$ ), which feeds into the motor programs (blocks) of attachment or surfeit behavior, depending on the sign of $\mathrm{App}_{\mathrm{Sec}}$. If the latter is positive, attachment behavior will be facilitated and surfeit behavior suppressed (open and solid arrowhead, respectively). If $\mathrm{ApP}_{\mathrm{Sec}}$ becomes negative, the attachment program is inactivated and the surfeit reaction disinhibited. 
appetence for security is equivalent to the difference between the infant's dependency and the feeling of security engendered by the mother.

I pointed out earlier that the incentive vector $\left(\mathrm{Inc}_{\mathrm{Sec}}\right.$ ) should also increase with proximity according to figure $2 c$. Hence, the (scalar) magnitude of this vector (i.e., the attractive valence of the attachment object in its given distance) and the amount of security experienced by the infant in the same situation may tentatively be regarded as equal. Figure 3 implies this by bifurcating a single variable into $\mathrm{Sec}$ and $\mathrm{Inc}_{\mathrm{Sec}}$.

Figure 4 illustrates the interdependence of the four hypothetical constructs of the security system, plotted against distance, in some typical situations.

Appetence behavior.-The block named appetence behavior in figure 3 is connected with the appetence component alone; it is therefore activated according to the dash-dot curves in figure 4. Although approaching an attachment object is also "appetent" in ethological terminology (Craig 1918), I shall speak here of "appetence behavior" in a more restricted sense. The term is meant to denote all kinds of instrumental behavior carried out under high drive pressure that cannot find a proper outlet because of insufficient stimulus conditions. If the dash-dot curve lies on the positive side of the abscissa, appetence behavior could, for example, appear in the form of distress calls, as assumed in figure 2. Some other forms of appetence will be discussed below.

Motor integration.-Since the programs for attachment, surfeit, and appetence behaviors are to some extent conflicting, they must be integrated-by suppression of the weaker in favor of the stronger, by alternation, or by some form of compromise. This is symbolized in figure 3 by the block named motor integration. In this part of the system, highly complex behavioral programming is very likely to occur. However, the present model is not meant to go into details in this respect. Plain linear superposition of all locomotor vectors involved will be assumed for the time being.

\section{The Arousal System}

Novelty.-As the next step, we must understand how and where fear fits into the pat- tern. I have already discussed the concept of distress; but, although this certainly has close connections with fear, the two must nevertheless be distinguished.

Throughout his life, an animal is incessantly surrounded by objects, live or inanimate, with which it must try to cope by properly responding to, cooperating with, or obviating their activities. The behavioral patterns employed by the animal for these purposes either can be preprogrammed by inheritance and previous learning, being triggered bluntly at the sight of fixed perceptual cues, or must be arrived at by means of ad hoc processing of information about the specific properties of the situation. In lower animals and young infants, the second type of response is seldom available, but it grows more important with ontogenetic and phylogenetic development.

The availability of these two forms of response depends likewise on the objects involved. Some objects tend to perform in a highly predictable manner; thus the individual can afford to react to them in a stereotyped way. An approaching predator or a child crying are examples of unambiguous objects that can be responded to without hesitation, provided the organism has the appropriate detectors to recognize them. But most objects are not so easily identifiable, and frequently the stimulus configuration received on the sensory screen does not even allow a vague guess about what is going to happen next. This effect is called stimulus novelty.

Relevancy.-In such a situation, the animal is temporarily at a loss about which one of his behavioral programs to release. Of course, he might block them all, not doing anything until things have cleared up. But this behavior does not seem to be advisable if the stimulus configuration, albeit unidentifiable, nevertheless is relevant. The concept of relevancy refers to the justified expectation that something very pertinent for the organism is likely to happen soon. A sudden change in the stimulus field, for instance, or cues showing that an object is looming in close proximity or quickly approaching, are stimulus situations in which it would probably be unwise to "wait and see."

Fear.-The better strategy, without doubt, is to behave like the professional photojournalist in an emergency - with no time to adjust his camera, he ventures to snap a quick shot, 


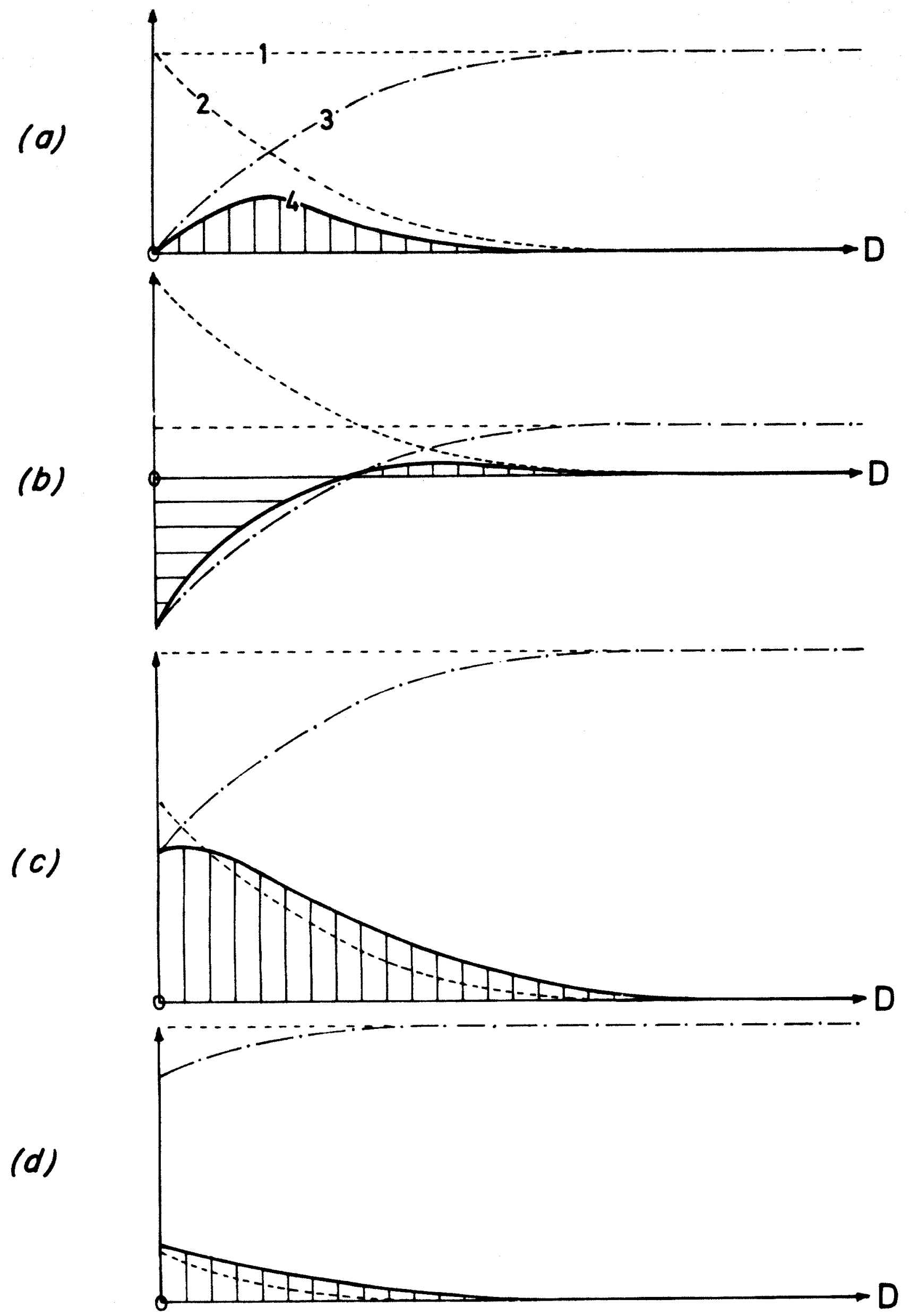

Frc. 4. - Some hypothetical variables of the security system (fig. 3) or the arousal system (fig. 5; variables in brackets) plotted against distance $(D)$. Abscissa as in fig. 2 . Ordinate: dashed horizontal lines $(1)=$ dependency [enterprise]; dashed curves (2) $=\operatorname{Inc}_{\text {Sec }}\left[\operatorname{Inc}_{\mathrm{Ar}}\right.$; dash-dot curves (3) $=$ App $_{\mathrm{Sec}}$ $\left[A_{A p p_{A r}}\right]$; solid curves $(4)=I m p_{S e c}\left(I_{m p}\right)$. Vertical hatching = likelihood and intensity of affiliative [exploratory] approach; horizontal hatching $=$ likelihood and intensity of surfeited [fearful] withdrawal. Situations: $a$, Moderate dependency [enterprise], adequate object, yielding approach response according to fig. 2b. Appetence (curve 3) strong only at high distance from object. b, Low dependency [enterprise], 
having preset the $f$-number and the exposure time to values most likely to be suitable for an average situation. Of course, this principle applies only when, among the set of available response programs, there is one that would be most likely, a priori, to optimize the animal's survival chances in the face of a relevant but novel object. Apparently the response of fear, originally developed as a means of avoiding harmful encounters, is exactly such an emergency measure, since it would cause the animal to withdraw from the object to a distance great enough to leave time to select a more suitable response.

Exploration.-The rationale of the foregoing strategy, however, is counterbalanced by still another requirement. To eventually produce the right response, the organism has to ascertain information about the object concerned. This, in turn, implies that the object has to be attentively observed, cautiously approached, and even carefully manipulated. Otherwise, novelty would remain unassimilated and would accumulate as a hindrance to competence. Thus, novel objects engender conflicts between two opposing responses.

Enterprise.-Everyday experience shows that individuals differ considerably in the way they resolve such conflicts. Certainly, the stimulus situation plays an important role. A very novel and highly relevant situation will be more likely to release fear than one that is already somewhat familiar and evokes a less dramatic impression. But even in similar contexts, the behavior of the same individual toward relevant novelty can vary considerably in different moods and different stages of development. In common language, an individual who is more likely to explore a novel situation than to withdraw from it is called "enterprising." This expression, which has already been used in developmental psychology (Rheingold 1973), is subsequently defined as denoting an internal reference variable controlling this likelihood.

Arousal.-It has become customary to use the hypothetical construct arousal in connection with the phenomena in question (Ber- lyne 1960; Mason 1965, 1971, 1973). Although the usage of this term in psychological literature is somewhat ambiguous, the general understanding of the concept appears to be that of a homeostatic variable of unknown physiological nature, which engenders fear responses when it exceeds a certain optimum level but instigates exploration when it falls short of that level. In the present model, this optimum level is controlled by the reference variable "enterprise" introduced above. Figure 5 presents this connection in the form of a control chart. Arousal itself, according to the foregoing consideration, is assumed to be generated by the relevance and the novelty of the situation. If one of these components is reduced to zero-that is, if the object is entirely familiar or entirely irrelevant-it will not arouse at all; therefore, the interaction of the two components is understood to be multiplicative.

Parallels to the security system.-The remainder of figure 5 closely resembles figure 3 , with security being replaced by arousal, dependency by enterprise, and the antagonism of attachment and surfeit by the antagonism of exploration and fear. Only the detector system is subdivided, with a special detector sensing relevancy (Det Rel ) and another $\left(\operatorname{Det}_{N}\right.$ ) assessing the degree of novelty by checking with the engram material stored in memory.

As in the case of attachment behavior, and for analogous reasons, a magnet-type and an elastic-band-type attraction by novel objects are distinguished in the model. The magnet-type effect can most easily be understood in terms of relevancy, since a nearby object is obviously more relevant than a distant one. Thus, if the organism has a lack of arousal because of a high enterprise level, a novel object becomes an even better lure when it is approached. Whether and how the novelty component itself varies with distance is intuitively less clear; this will be discussed elsewhere (Bischof 1975b).

The stated analogies between figures 3 and 5 allow one to interpret the curves in

adequate object. Close proximity of object has a repulsive effect due to surfeit [fear]. Only at sufficiently high distance will there be a weak positive interest in the object. $c$, Very high dependency [enterprise], adequate object. Approach response as in fig. 2c. High appetence, which is only reduced but does not vanish in close proximity to object. $d$, High dependency [enterprise], insufficient object (i.e., negligent care giver [boring stranger]). Reluctant approach according to fig. $2 c$. High amount of appetence peristing irrespective of distance from object. 


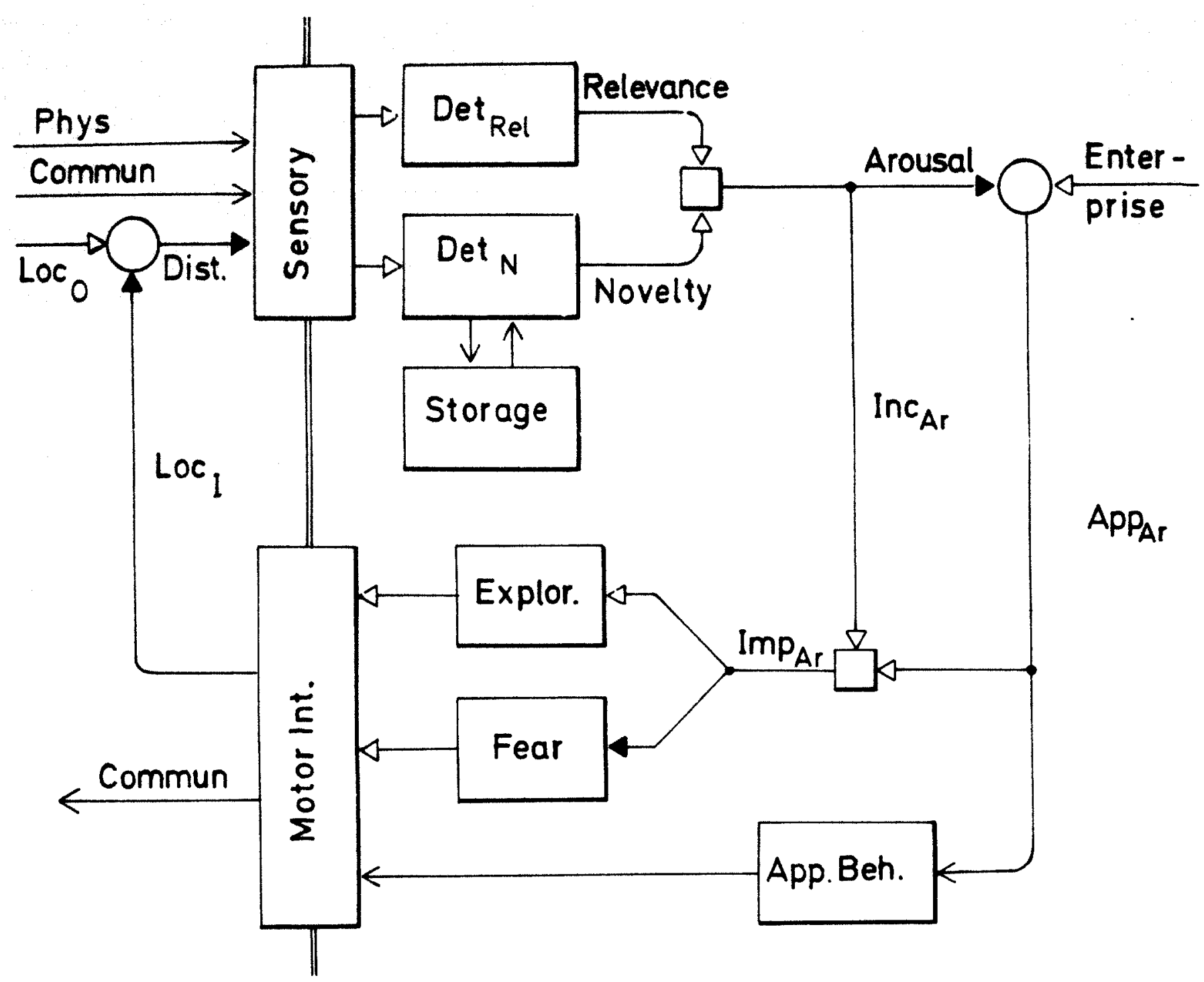

Frc. 5.-Arousal system. Interpretation analogous to that of fig. 3. Subscript $M$ (mother) replaced by $O$ (object).

figure 4 in terms of the arousal system as well. For instance, figure $4 b$ can account for the case of a stranger encountered in a state of low enterprise, who releases a flight response that will come to a halt only at a sufficiently safe distance and then give way to a mild tendency to approach. This approach may appear in the form of attentive observation, or even of cautious following if the object happens to move away.

\section{The Compound System}

Maturational delay of arousal.-Having developed the structures of the security and arousal systems separately, one may ask how they can be conceived of together.

Before the mother becomes an attachment object, she is, of necessity, a novel stimulus. She is also a relevant stimulus, since often the only requirement for filial imprinting is that the object be proximal, conspicuous, and interacting with the infant, and these are all cues of relevancy. In terms of the arousal model, however, such a novel and relevant object should produce an intensive fear response which would interfere with the formation of attachment. This fatal effect could be avoided by the mother emitting special fearinhibiting signals to which the infant has been genetically preprogrammed to react. Imprinting is often facilitated considerably if the care giver responds to the infant's distress calls by brief vocalizations, which might be indicative of a mechanism of this kind. The overall evidence, however, indicates that the problem is solved in another way. The phenomenon described by Spitz (1965) as "eight-month anxiety," which has analogues in many social animals (Bateson 1966; Hess 1959; Scott 1962), can best be understood by the assumption that there is a maturational delay between the security and the arousal systems, with at least some essential parts of the latter developing late enough to allow previous familiarization with the mother to occur. (In some cases of childhood autism the maturation of arousal 
may be so precocious that it interferes with this familiarization.)

At this juncture, it is easy to see the necessity of a clear-cut distinction between "distress" and "fear," as discussed earlier. Distress belongs to the security system, fear to the arousal system. After maturation of the latter, to be sure, distress and fear become highly correlated; but in the preceding period there is mainly distress (when separated from the mother) and only rudiments of fear (when confronted with a stranger).

Detector combination.-Attachment relationships could then be understood to build up in the following way. Filial approach behavior will first be elicited by any conspicuous, interacting, and proximal stimulus (i.e., by any relevant stimulus). The ensuing close proximity will provide thorough familiarization with this attachment object, and after maturation of the arousal system this familiarity will inhibit arousal and facilitate security. Cues of relevancy will continue to enhance the security response as well, since, everything else being equal, a familiar object can be assumed to provide more security when it is close and interacting than when it is far away and not taking notice.

This means that both detector mechanisms appearing in figure 5 as parts of the arousal system can also be considered as working in the security system. Familiarity and novelty are complementary, and any gauge capable of assessing one must also measure the other. We can therefore conceive of a double output of the novelty detector, both branches conveying essentially the same information but one denoting the degree of familiarity and feeding into the security system while the other denotes novelty and activates arousal.

Stimulus interaction.-Figure 6 illustrates how the two systems depicted in figures 3 and 5 can thus be integrated. Without additional assumptions, this compound model accounts for the well-established observation that fearevoking stimuli are likely to intensify attach-

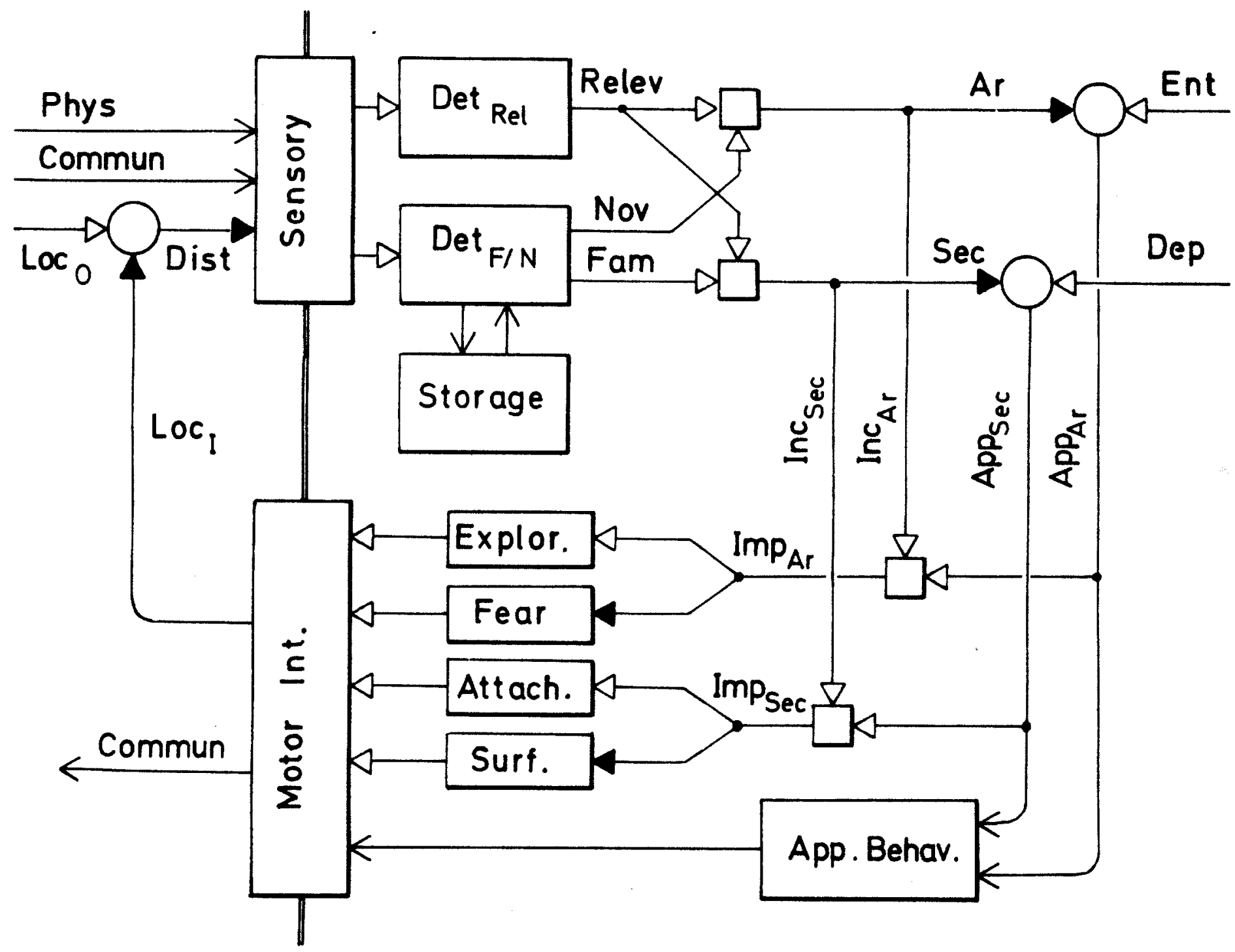

Fig. 6.-Final version of the model. $\operatorname{Det}_{F / N}=$ detector of familiarity and novelty. All other symbols as in figs. 3 and 5. For further explanation see text. 


\section{Child Development}

ment behavior toward familiar objects, whereas the proximity of attachment partners, conversely, renders the individual more audacious to explore novel objects. This is so since the introduction of any familiar stimulus reduces the novelty-familiarity ratio, thus both contributing to security and lowering the net arousal level. The same applies to the opposite situation in which an additional novel stimulus is introduced.

Appetence behavior.-The appetence behavior system is put into action, according to the model, by an excessive deviation of security or arousal from their reference variables in either direction. Such a deviation will normally occur in a state of "frustration," that is, in situations where the incentive vectors vanish because of suitable objects being undetectable or inaccessible. The appetence response can consist of three main forms of instrumental activity: searching behavior, aggressive behavior, and supplicative behavior.

Searching behavior, in its most primitive form, may be confined to unorganized locomotor unrest, possibly interspersed with fragments of irrelevant behavioral patterns known as "displacement activities." In more advanced stages of behavioral development, experience and intelligence play an increasing role in organizing searching activities. In ethology, searching is considered the most typical form of appetent behavior, whereas psychologists and psychiatrists tend to lay more emphasis on the appetent character of aggression and supplication (Hamburg, Hamburg, \& Barchas, in press).

Aggressive behavior, if thus considered as an instrumental activity in a state of frustrated appetence, ought to come as a possible concomitant or substitute of any one of the four motivational programs provided by the model. This is indeed the case. Aggression may come as an exaggeration of exploratory intentions if arousal is deficient, as in juvenile play fighting or in the more serious instances of adolescent violence arising from boredom. It may be the inversion of fear in the wellknown "critical reaction" occurring when escape is blocked or when excessive arousal is caused by a pathologically low level of enterprise. In the form of hate, or of infantile naughtiness (especially when following separation), aggression may be the shadow of love. And it can certainly be the expression of surfeit when withdrawal is barred.
A third possible reaction to frustrated appetence is supplicative behavior. This, too, may occur in all four situations of disequilibrium provided by the model. If security is deficient, supplicative behavior will consist in the emission of distress calls, in symptoms of depression, or, if the attachment object is present but unresponsive, in exaggerated seeking for attention and support. In a state of excessive arousal, supplicative appetence will appear in the form of submissive behavior and may also underlie what psychoanalysts have described as "identification with the aggressor." And that supplicative activities may, paradoxically enough, occur even in states of appetence for more arousal and less security is amusingly illustrated by the true story of a youngster seriously demanding, "Mom, get me a taxi, I wanna run away from home!"

Adaptation.-If, in a state of disequilibrium, neither the specific locomotor responses of attachment, surfeit, exploration, and fear nor the three unspecific appetence activities of searching, aggression, and supplication lead to tension release, the individual is forced to adapt to the situation. This possibility has not been incorporated in figure 6 in order to avoid undue complexity of the chart; but it is important enough to be at least mentioned. Adaptation can occur, in terms of the model, at two main points.

One way of adapting to an unbalanced social situation is to adjust the internal reference levels of dependency and enterprise. $\mathrm{Ob}-$ viously, an individual chronically overaroused and lacking security because of continuous negligence or early loss of the care giver may, after a while, sheathe himself in a forced attitude of independence and recklessness. In complementary fashion, extreme dependency and anxiety may be developed as a capitulation to the continuous surfeit-loaded atmosphere of an omnipresent, overprotective mother.

The second possible site of adaptation is the familiarity detector. One way to escape the stress of incessant exposure to unfamiliar surrounds is to bias the familiarity detector in the direction of familiarity. The price to be paid here is a reduced discrimination on the familiarity scale and a superficially welladjusted, yet noncommittal and slightly indiscreet, intimacy with everybody. A bias of the familiarity detector in the opposite direction 
could result in alienation and general paranoiac mistrust. The role of dominant, overprotective mothers in the pathogenesis of some forms of psychosis gains some intuitive plausibility under this viewpoint.

Other detectors.-An additional word should be said about the role played by other types of detectors in the foregoing system which are likely to support or counteract the effects of relevancy, familiarity, or novelty.

Most important in this respect are detectors sensitive for certain types of conspecifics. Conspecifics in general often simply appear to contribute to relevancy, since many animals prefer members of their own species as objects of both attachment and exploration.

Moreover, there seems to be a widespread tendency in the animal kingdom to develop detectors capable of identifying children. Such mechanisms were discovered and described by Lorenz (1943), under the name of "Kindchen-Schema" ("baby-detector"), as a releasing mechanism for the response of maternal care giving. There is reason to believe, moreover, that these detectors may mature as early as in childhood. Children are conspecifics who are not likely to do one any serious harm; one can, therefore, afford to reckon them as harmless even if unfamiliar, thus excluding them from the set of arousal-generating stimuli. Moreover, chances are high that children encountered early in development are attached to the same group of care-giving adults as the individual himself; therefore, they can serve as a cue leading to security when the mother happens to be out of sight. Child detectors could therefore conceivably function as a pure source of "familiarity" signals increasing the security level and attenuating arousal.

Finally, to prevent homosexuality and hybridization, the animal should be endowed with sex detectors that allow it to identify male and female conspecifics. This detector system, to be sure, needs to be available only in mature animals; but again it might develop earlier, as in the case of sexual imprinting. The output of such detectors may contribute to the security and arousal levels in a specific way, thus accounting for the different forms of attachment and fear shown by infants toward individuals of different sex, irrespective of familiarity.

\section{Illustration}

Realization of the model.-The previous considerations would be unsatisfactory unless illustrated by at least a simple quantitative example. Therefore, the prediction of the model was computed for the case of an individual in two different stages of development, confronted in a neutral room with his mother and with a stranger.

The mathematical realization of the model structure is too involved to discuss here. The simulation is based on figure 6, with appetence behavior represented by motor unrest. Aggression, supplication, and adaptation are not incorporated in the simulation program. Certain complications are unavoidable as soon as we introduce a two-dimensional space in which the child can move about or present the child with more than one object at the same time. A detailed description of the simulation procedure will be published elsewhere (Bischof $1975 b)$. At present, I shall confine myself to the results.

Simulation of infancy.-Figure 7 shows the model simulation of an infant's behavior at an early age but after full maturation of the arousal system. I assume the dependency level to be high and the enterprise level to be low at this stage. When exposed to the mother after having been left alone (fig. 7a), the infant will hurry to her and quietly remain there. If a stranger is introduced (fig. $7 b$ ), the child will come as close to the mother as possible and will even try to "drag her away" from the stranger by moving still further to the left and showing slight signs of uneasiness, which might prompt a normal mother to follow him. If the mother leaves the infant alone with the stranger (fig. $7 c$ ), there will be a tendency to approach the latter, less quickly, though, and less close. In the stranger's proximity there will remain a considerable amount of appetence unrest due to fear and distress.

Simulation of puberty.-A quite different behavioral pattern ensues in the same situation when the child has reached a later stage of development, for instance, adolescence. I assume that this stage is characterized by a partial decay of dependency and an increase of enterprise. If a juvenile in this condition is exposed to the mother alone (fig. 8a), he will still approach her, albeit reluctantly, but the approach will come to a halt early enough to make sure that no close contact will ensue. 
(a)

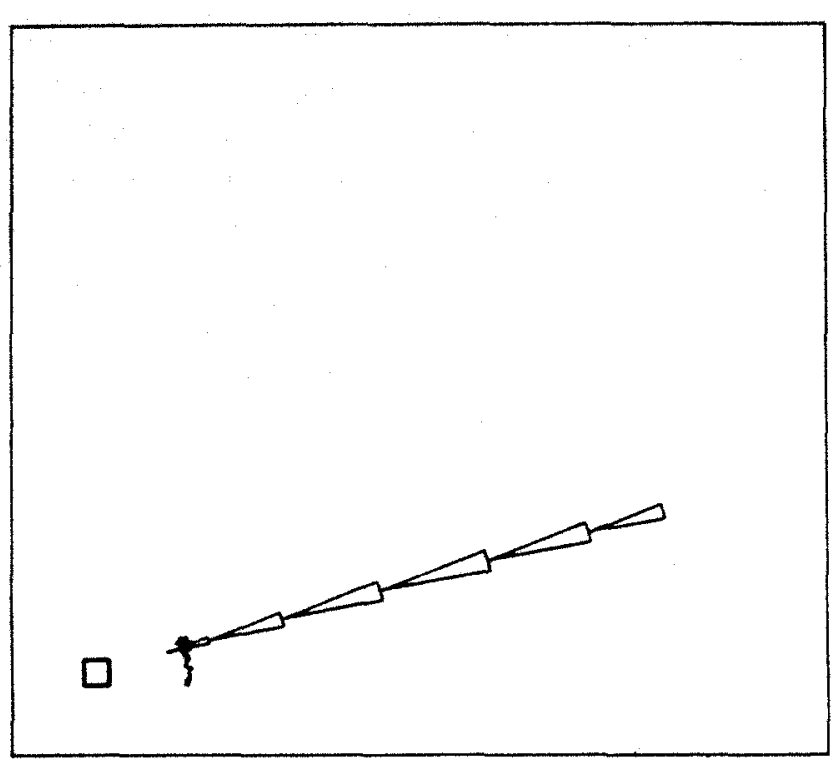

(b)

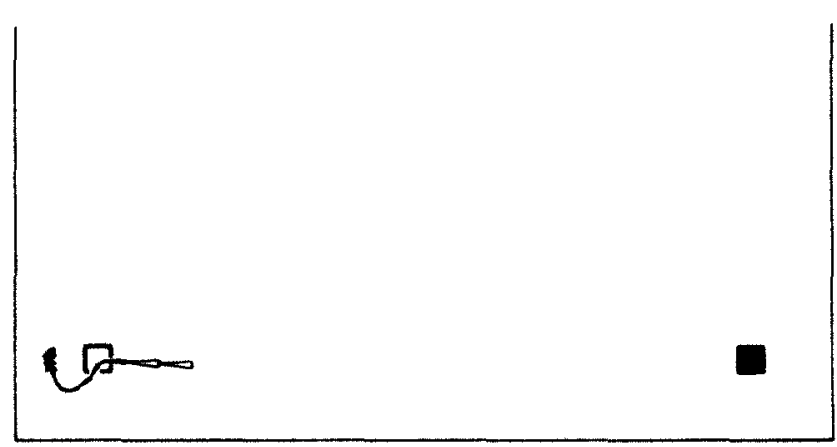

(c)

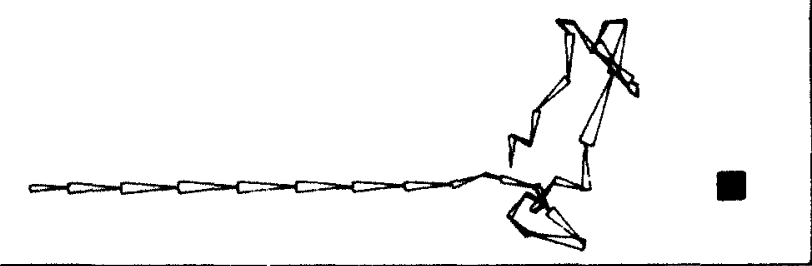

Fic. 7.-Locomotor behavior of infant in state of high dependency and low enterprise. Computer simulation according to model in fig. 6. Arrows $=$ locomotor steps, computed for 100 iterations. Length of arrow denotes momentary speed of movement. Conditions: $a$, Mother alone exposed to infant. $b$, Stranger entering. $c$, Mother leaving child alone with stranger. Initial position of infant in $a$, arbitrary; in $b$ and $c$, identical with final position of preceding situation. Upper half of room skipped in $b$ and $c$ to save space.

Again, there will be a high level of motor unrest, showing that there are unresolved appetence tensions present in the youngster. The nature of these tensions is revealed as soon as we introduce a stranger (fig. $8 b$ ), who is at once approached cautiously, but steadily, up to a state of closest possible contact. Removing the mother (fig. 8c) will now make little difference-she is no longer of crucial importance. Only a slight increase in tension can be seen in the youngster when left quite alone with the stranger: the latter is still a bit too unfamiliar to prevent traces of fear being blended into the attachment, but the intimacy persists and will increasingly mitigate the remaining uneasiness as time goes on.

It is a noteworthy feature of the model that, in the condition of figure $8 b$, a further increase of dependency would not draw the juvenile back into the arms of his mother. In fact, as soon as enterprise has been high enough to cause the individual to approach the stranger beyond a certain equilibrial point specified by the mathematical structure of the model, any further increase of dependency will only intensify the attachment to the stranger, driving the individual even farther away from his mother. This prediction reveals interesting perspectives on the possible motivational dynamics underlying the attachment between mates, which is enigmatic because it resem-

(a)
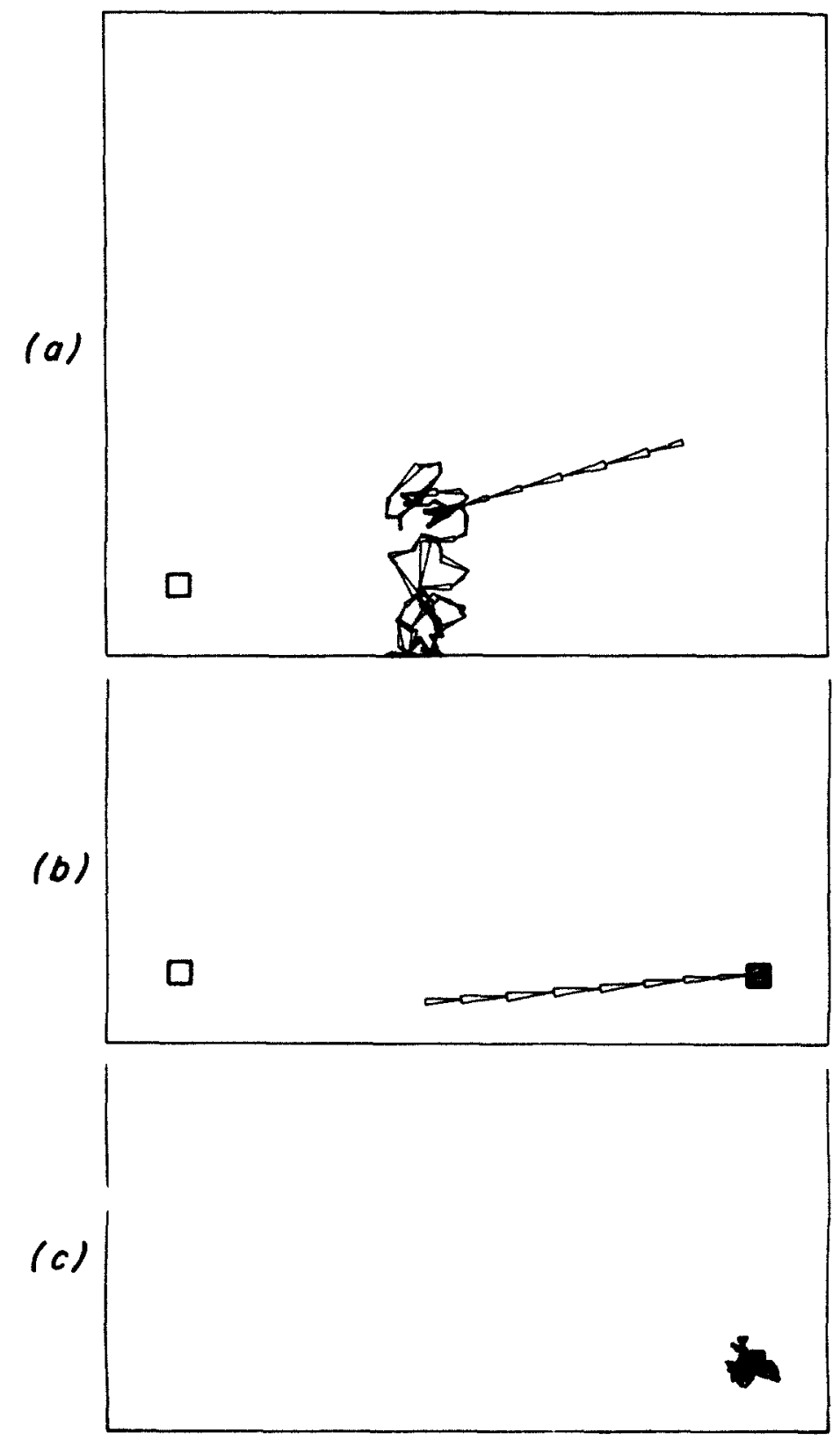

Fig. 8.-Locomotor behavior of adolescent in state of medium dependency and medium enterprise. Explanation as in fig. 7. 
bles in many respects the attachment of a child to his parents and still excludes the natural parent as a possible object.

Empirical support.-Locomotor tracks as depicted in figure 7 can indeed be obtained in experiments with animal infants. Figure 9 shows the locomotor behavior of four 3-weekold graylag goslings when released from an opaque box on a large, even sand arena in the presence of their own (human) foster mother $(a)$ or of a human stranger $(b)$. The correspondence with the model prediction as depicted in figures $7 a$ and $c$ is quite reasonable. Note specifically that (1) the approach velocity often shows the characteristic course of figure $2 b ;(2)$ the mother object is approached with higher speed; (3) the mother object is contacted more closely than the stranger (the case in line 3 of fig. $9 b$ is no exception, since the short final portion of the trace which seems to lead close to the stranger is actually twofold and indicates an approach followed by a withdrawal); (4) after the approach to the stranger there remains a greater amount of motor unrest, as indicated by the random excursions; and (5) the final random excursions often have a semicircular form, which is also characteristic of the simulated traces in figures 7 and 8.

\section{Conclusion}

At the beginning of this paper, Bowlby's (1971) theory was introduced as a "first step" toward a systems analysis of early infant social motivation. Admittedly, the model described in our study cannot claim to be more than a second step-and it is hard to know how many such steps will have to follow. Future elaboration will have to incorporate certain salient facets of social behavior that are neglected in the present model-for instance, smiling and laughing, the coy response described by Bretherton and Ainsworth (1974), the phenomenon of shame, the systems of sexual and maternal motivation, the readiness to attract as well as to pay attention (Chance 1967).

Furthermore, there seems to exist a social control of the variables labeled "dependency" and "enterprise" in the model, and this control may be essentially connected with ranking order. So far, I have basically considered maturational influences that increase enterprise and decrease dependency during ontogeny. But it is quite clear that experiential influences also come into play. Some of them I outlined briefly in the section on "Adaptation" above. There is, further, all the evidence supporting Erikson's (1950) theory of "basic trust" or Klein's (1948) concept of "introjection of the good object" (cf. Bowlby 1971, p. 402). These authors assume that continuous exposure to a properly behaving care giver (hence, a persistently high level of security in early childhood) will, by means of slow but steady internal accumulation, reduce dependency to a certain degree, thus rendering the individual able to undergo temporary shortages of external reassurance without affective breakdown. This could indicate a heavily damped negative influence of security on dependency; and there may be many more cross-connections of this kind.

A special difficulty arises whenever the model is applied to higher animals or even man. Here, unlike the case of graylag geese, the basic dimensions of interindividual distance and locomotion can no longer be expected to be always measurable in meters. Instead, imaginary Lewinian spaces often substitute for physical space. This, too, remains to be incorporated in the model.

Last, but not least, there is a formal cue indicating that the model as it stands is structurally imperfect: the parallelism between the security and the arousal systems appears to be all too symmetrical. Systems which have gradually evolved under the sway of natural selection are seldom of consummate order, and, if they are, they show a functional order, not the order of intellectual esthetics. The laws which allocate every structural element of an organism to its proper place are laws of purposiveness rather than of symmetry. Symmetry is what our gestalt perception quite often erroneously projects on such systems as long as the power of our knowledge is too weak to resist.

In summary, the model presented cannot claim to be more than a preliminary working hypothesis, and we ought not to be surprised if changes necessary in the course of future elaboration modify it substantially. However, such an eventuality would not render the attempt worthless. The systems approach has the advantage of allowing and encouraging changes and providing a solid basis for every new step. This is more than the traditional verbal theories that pervade our field of research have to offer. 

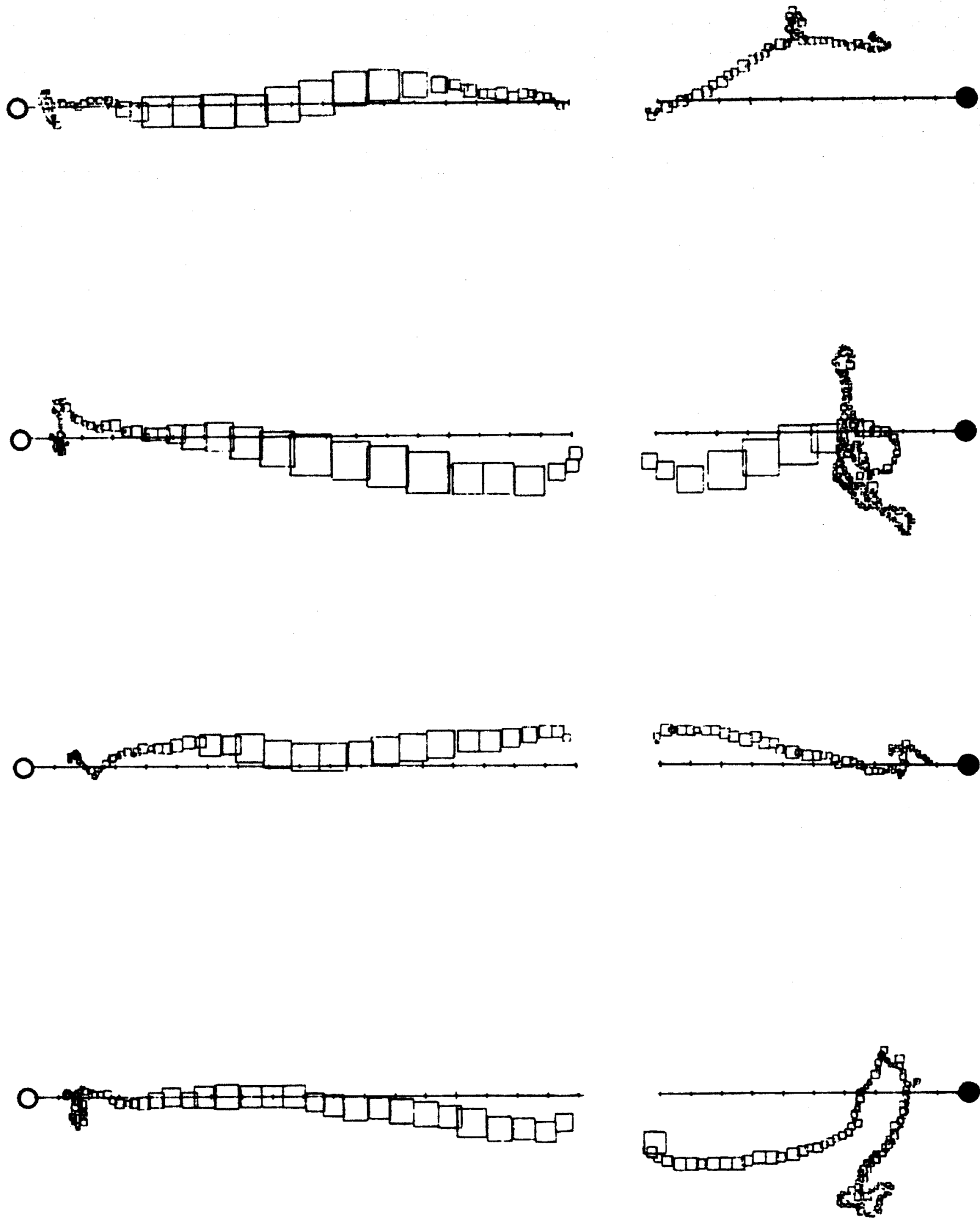

(a)

(b)

Fic. 9.-Empirical data in accordance with model predictions: traces of approach behavior of four graylag goslings aged approximately 3 weeks when exposed to $a$, their human imprinting object (open circle) and $b, a$ human object only remotely familiar ( solid circle). Side-length of square denotes momentary velocity for every half-second interval; center of square indicates momentary position. Grid line shows shortest possible connection between starting point (right end of line in $a$, left end in $b$ ) and target object. Scale $=1 \mathrm{~m}$. Initial distance to target object: $a, 18 \mathrm{~m} ; b, 10 \mathrm{~m}$. Examples taken from a pilot study performed to develop and test the experimental setup and recording technique. 


\section{Norbert Bischof 817}

\section{References}

Ainsworth, M. D. S., \& Wittig, B. A. Attachment and exploratory behavior of one-year-olds in a strange situation. In B. M. Foss (Ed.), Determinants of infant behavior. Vol. 4. New York: Barnes \& Noble, 1969.

Bateson, P. P. G. The characteristics and context of imprinting. Biological Review, 1966, 41, 177-220.

Berlyne, D. E. Conflict, arousal, and curiosity. New York: McGraw-Hill, 1960.

Bischof, $N$. The biological foundations of the incest taboo. Social Science Information, 1972, 11, 7-36. (a)

Bischof, N. Inzuchtbarrieren in Säugetiersozietäten. Homo, 1972, 23, 330-351.(b)

Bischof, N. Comparative ethology of incest avoidance. In R. Fox (Ed.), Biosocial anthropology. Association of Social Anthropologist's Studies, No. 1. London: Malaby, 1975.(a)

Bischof, N. A mathematical model of social motivation. Unpublished manuscript, Max Planck Institute for Behavioral Physiology, 1975.(b)

Bowlby, J. Attachment and loss. Vol. 1. Attachment. Harmondsworth, Middlesex: Penguin, 1971.

Bowlby, J. Attachment theory, separation anxiety and mourning. In D. A. Hamburg \& H. K. Brodie (Eds.), The American handbook of psychiatry. Vol. 6. New York: Basic, in press.

Bretherton, I., \& Ainsworth, M. D. S. Responses of one-year-olds to a stranger in a strange situation. In M. Lewis \& L. Rosenblum (Eds.), The origins of fear. New York: Wiley, 1974.

Chance, M. R. A. Attention structures as the basis of primate rank orders. Man, 1967, 2, 503518.

Craig, W. Appetites and aversions as constituents of instincts. Biological Bulletin, 1918, 34, 91107.

Erikson, E. H. Childhood and society. New York: Norton, 1950.

Gewirtz, J. L. (Ed.) Attachment and dependency. Washington, D.C.: Winston, 1972.
Hamburg, D. A.; Hamburg, B. A.; \& Barchas, J. D. Anger and depression in perspective of behavioral biology. In L. Levi (Ed.), Parameters of emotion. New York: Raven, in press.

Hess, E. H. The relationship between imprinting and motivation. In M. R. Jones (Ed.), Nebraska symposium on motivation. Lincoln: University of Nebraska Press, 1959.

Klein, M. Contributions to psycho-analysis, 19211945. London: Hogarth, 1948.

Lorenz, K. Die angeborenen Formen möglicher Erfahrung. Zeitschrift für Tierpsychologie, $1943,5,235-409$.

Mason, W. A. Determinants of social behavior in young chimpanzees. In A. M. Schrier, H. F. Harlow, \& F. Stollnitz (Eds.), Behavior of nonhuman primates. Vol. 2. New York: Academic Press, 1965.

Mason, W. A. Motivational factors in psychosocial development. In W. J. Arnold \& M. M. Page (Eds.), Nebraska symposium on motivation. Lincoln: University of Nebraska Press, 1971.

Mason, W. A. Regulatory functions of arousal in primate psychosocial development. In C. $\mathbf{R}$. Carpenter (Ed.), Behavioral regulators of behavior in primates. Lewisburg, Pa.: Bucknell University Press, 1973.

Rheingold, $H$. L. Independent behavior of the human infant. In A. D. Pick (Ed.), Minnesota symposia on child psychology. Vol. 7. Minneapolis: University of Minnesota Press, 1973.

Rheingold, H. L., \& Eckerman, C. O. The infant separates himself from his mother. Science, $1970,168,78-83$.

Scott, J. P. Critical periods in behavioral development. Science, 1962, 138, 949-958.

Sears, R. R.; Rau, L.; \& Alpert, R. Identification and child rearing. London: Tavistock, 1966.

Sears, R. R.; Whiting, J. W. M.; Nowlis, V.; \& Sears, P. S. Some child-rearing antecedents of aggression and dependency in young children. Genetic Psychology Monographs, 1853, 47, 135-236.

Spitz, R. A. The first year of life. New York: International Universities Press, 1965. 
This document is a scanned copy of a printed document. No warranty is given about the accuracy of the copy. Users should refer to the original published version of the material. 